\title{
Analysis and Improvement of Routing Protocol LEACH
}

\author{
Rahul Rajpal \\ Engineer, B.Tech., \\ Information Technology \\ GGSIPU, New Delhi
}

\begin{abstract}
Energy efficient design and latest wireless technologies have enabled new exciting applications for wireless devices. The Sensor networks have provided solutions to many problems in the areas of infrastructure, disaster management, military sensing, traffic management etc. and have become one of the most fascinating areas of research in the past few years. These applications require high performance on the network but Wireless devices are often having limited resources because nodes are operated by battery. This creates problem due to limited bandwidth \& makes transfer error prone. A wireless sensor network which consists of a group of sensor nodes enables the monitoring of a variety of environments for applications. In WSNs, it is very vital for network to operate for a long time. In this case, data fusion helps to reduce the amount of data exchanged between sensor nodes and base station. The LEACH (Low Energy Adaptive Clustering Hierarchy) protocol is a well-designed solution to this problem. This research paper discusses LEACH protocol is discussed.
\end{abstract}

\section{General Terms}

Broadcasting, Routing, Wireless Radio Communication, Architecture, Distributed algorithm, Energy-efficiency, Hybrid Protocols, Clustering Protocols

\section{Keywords}

LEACH, Cluster-base, Data aggregation, TDMA, Steady State Phase, Set-up phase, Cluster-head advertisement

\section{INTRODUCTION}

In recent years we have seen growth of wireless devices including cellular phone, mobiles, laptops and personal digital assistants. The advances in the wireless technology are also one of the major motivations for the growth of the wireless devices or mobile computing. And for this, basically the Wireless Sensor Network should perform its functionality as long as possible.

Many routing protocols have been proposed for WSN [2][3] [4]. Some of them are cluster based. Two of the well-known hierarchical protocols are LEACH and PEGASIS as they show significant drop in the overall network energy over other alternatives present.

Hierarchical routing protocols are designed to reduce energy consumption by localizing communication within the cluster and aggregate data to reduce number of transmissions to the BS. Leach is considered as the most popular routing protocol that uses cluster based routing in order to reduce the energy consumption; following section describes LEACH protocol, its architecture, analysis \& its simulation.

\section{CLUSTER BASED ROUTING}

\subsection{Clustering and Broadcasting}

In WSNs, broadcasting is an important process for data transmission. Broadcasting is a process in which a source node transmits messages to all other nodes of the network Clustering is a method for Broadcasting.

LEACH (Low Energy Adaptive Clustering Hierarchy), a clustering-based protocol that utilizes randomized rotation of cluster heads to evenly allocate the energy load among the sensors in network. LEACH uses localized coordination to enable scalability and robustness for dynamic networks, and incorporates data fusion into the routing protocol to reduce the amount of data that must be sent to the base station.

\subsection{Working of Cluster based routing}

In clustering schemes, there are two kinds of nodes in one cluster, one cluster head $(\mathrm{CH})$ and several other cluster members (CMs). Cluster members gather data from the environment periodically and send the data to cluster heads. As shown in Figure 1, Cluster heads aggregate the data from their cluster members, and send the aggregated data to the base station (BS). There are two kinds of communications between cluster heads and the BS, single-hop communication and multi-hop communication. In multi-hop communication clustering algorithms, the energy consumption of cluster heads consists of the energy for receiving, aggregating and sending the data from their cluster members, known as intracluster energy consumption and the energy for forwarding data for their neighbor cluster heads known as inter-cluster energy consumption[11].

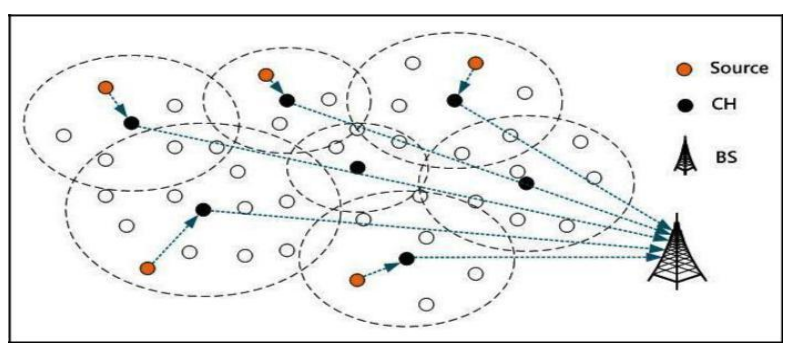

Fig.1 Clustering based routing in WSN

The main goal of cluster-based routing protocol is to efficiently maintain the energy consumption of sensor nodes by involving them in multi-hop communication within a cluster. Also, it uses data aggregation and data fusion in order to decrease the number of transmitted messages to the sink and transmission distance of sensor nodes.

\section{LEACH}

\subsection{About LEACH}

LEACH (Low Energy Adaptive Clustering Hierarchy) is a cluster-based protocol, which includes distributed cluster formation. LEACH selects few sensor nodes randomly as cluster-heads and rotates this role to evenly allocate the energy load among the sensors of network. In LEACH, the cluster-heads reduce data arriving from nodes that belong to the respective cluster, and send an aggregated packet to the BS so that total information transmitted to the BS decreases. 


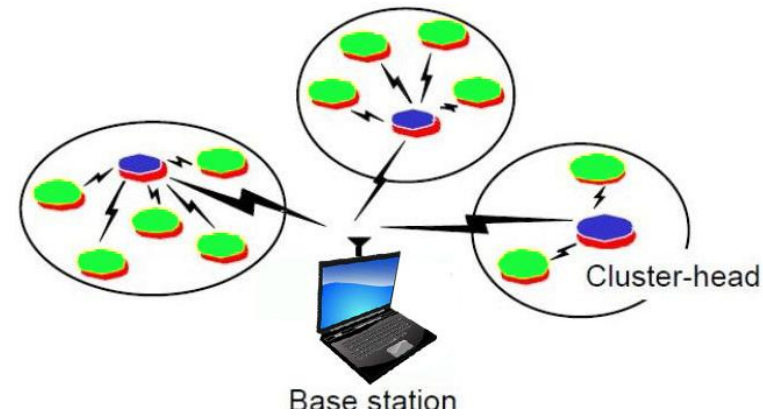

Fig.2 LEACH based formation with $\mathrm{BS}$ and $\mathrm{CH}$

\subsection{LEACH Protocol}

This protocol is divided into different rounds; each round consists of two phases:

i. $\quad$ Set-up Phase

ii. Steady Phase

\subsubsection{Set-Up Phase}

Set-up phase is further divided into 2 parts:

- Advertisement Phase

- Cluster Set-up Phase

LEACH forms clusters by using a distributed algorithm where nodes make autonomous decisions without any centralized control.

In the Advertisement Phase, $\mathrm{CHs}$ inform their neighborhood with an advertisement packet that they become CHs. Non-CH nodes pick the advertisement packet with the strongest received signal strength. Each node decides independent of other nodes if it will become a $\mathrm{CH}$ or not. This decision is made by looking into account when the node served as a $\mathrm{CH}$ for the last time (means the node that hasn't been a $\mathrm{CH}$ for long time is more likely to elect itself than nodes that have been a $\mathrm{CH}$ recently). This is done according to a threshold value, $\mathrm{T}(\mathrm{n})$. The threshold value depends upon the desired percentage to become a cluster-head- $p$, the current round $r$, and the set of nodes that have not become the cluster-head in the last $1 / p$ rounds, which is denoted by $\mathrm{G}$. Based on all messages received within the cluster, the $\mathrm{CH}$ creates a TDMA schedule, pick a CSMA code randomly, and broadcast the TDMA table to cluster members every node wanting to be the cluster-head chooses a value, between 0 and 1 . If this random number is less than the threshold value, $T$ (n), then the node becomes the cluster- head for the current round. Then each elected $\mathrm{CH}$ broadcasts an advertisement message to the rest of the nodes in the network to invite them to join their clusters. Based upon the strength of the advertisement signal, the noncluster head nodes decide to join the clusters. In the set-up phase, the cluster head nodes are randomly selected from all the sensor nodes and several clusters are constructed dynamically. Figure 3 represents flowchart that shows the procedure for the set-up phase.

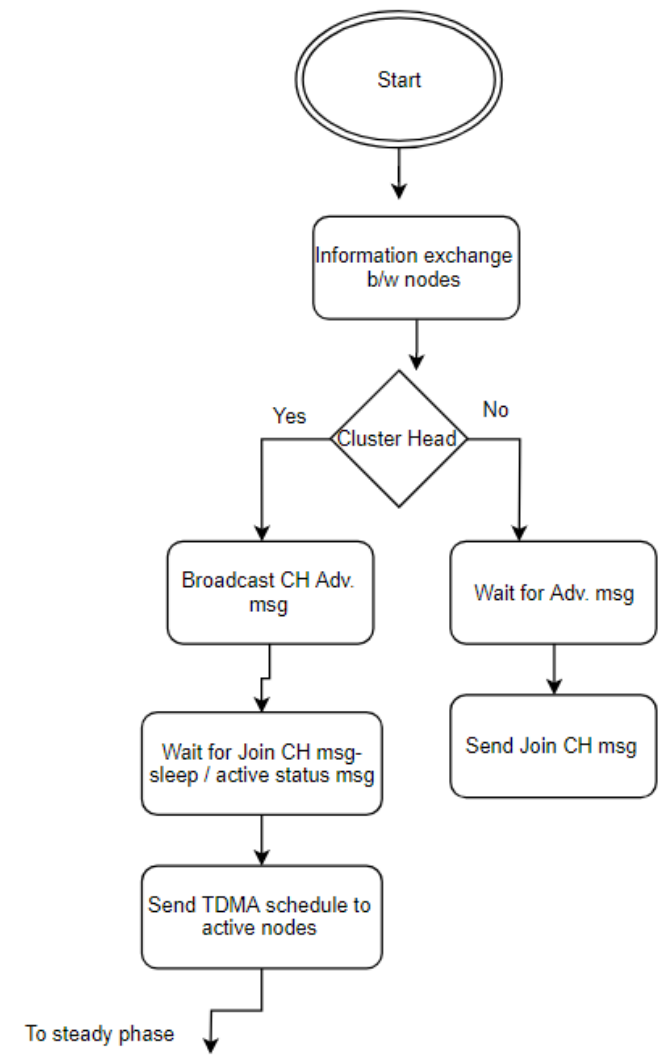

Fig.3 Flowchart of LEACH protocol in Set-up phase

\subsubsection{Steady Phase}

Steady phase is further divided into 2 parts:

- Schedule creation

- Data Transmission

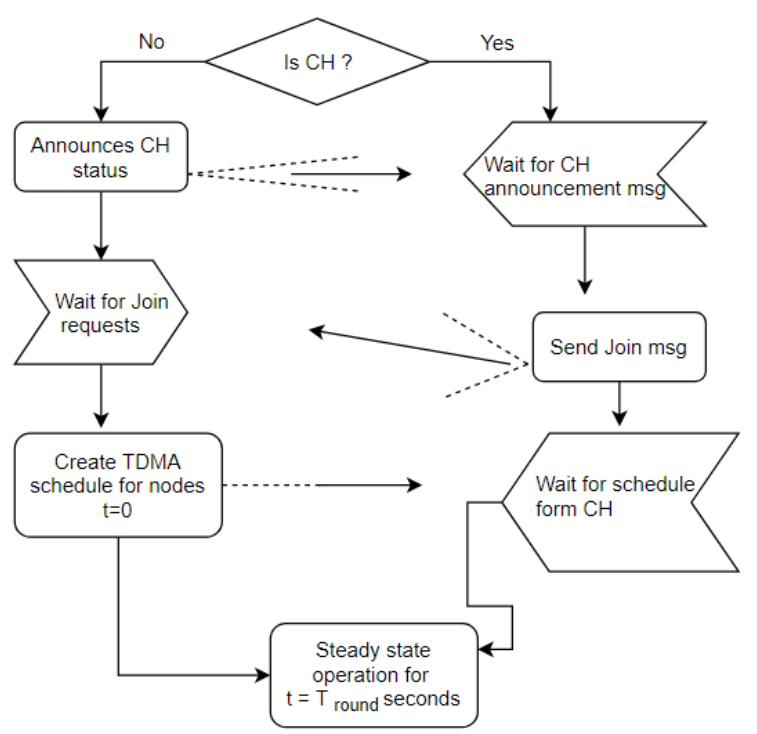

Fig.4 Flowchart of LEACH protocol in Steady phase

Once the nodes have elected themselves to be cluster heads the cluster head nodes must let all the other nodes in the network know that they have chosen this role for the current round. To do this each cluster head node broadcasts an advertisement message ADV using a non-persistent carrier sense multiple access CSMA. This message is a small message containing the nodes ID and a header that 
distinguishes this message as an announcement message. Each non cluster head node determines to which cluster it belongs by choosing the cluster head, that requires the minimum communication energy based on the received signal strength of the advertisement from each cluster head. After each node has decided to which cluster it belongs, it must inform the cluster head node that it will be a member of the cluster. Each node transmits a join request message (Join_REQ) back to the chosen cluster head using a non-persistent CSMA. After schedule creation sub phase, the $\mathrm{CH}$ knows the number of member nodes and their IDs. Based on all messages received within the cluster, the $\mathrm{CH}$ creates a TDMA schedule, pick a CSMA code randomly, and broadcast the TDMA table to cluster members. After that Data Transmission phase begins. Nodes send their data during their allocated TDMA slot to the $\mathrm{CH}$. This transmission uses a minimal amount of energy (chosen based on the received strength of the $\mathrm{CH}$ advertisement) the radio of each non- $\mathrm{CH}$ node can be turned off until the nodes allocated TDMA slot. This will minimize energy dissipation in nodes. Once all the data has been received from the cluster nodes, the $\mathrm{CH}$ aggregate these data and send it to the BS.

LEACH is able to perform local aggregation of data in each cluster to reduce the amount of data that transmitted to the base station.

\section{Proposed Routing Formula}

The Election of Cluster Head is Dependent upon a formula that takes into consideration the following parameters:

$$
\begin{array}{cl}
\text { i. } & \text { Number of rounds: } \mathrm{p} \\
\text { ii. } & \text { Threshold Value: } \mathrm{T}(\mathrm{n}) \\
\text { iii. } & \text { Current Round: } \mathrm{r} \\
\text { iv. } & \text { Unelected Nodes: } \mathrm{G}
\end{array}
$$

Existing formula:

$T(\mathbf{n})=\mathbf{p} /(\mathbf{1}-\mathbf{p} * \bmod (\mathbf{r}, \operatorname{round}(\mathbf{1} / \mathbf{p}))$

New proposed formula introduces another parameter that takes into account the distance between the "to be elected $\mathrm{CH}^{\prime}$ and the Base Station. The reason for selecting this parameter is that, distance between $\mathrm{CH}$ and BS is directly related to the transmission time and hence Energy Depleted, i.e. higher the distance, higher transmission time and energy. The following formula has been introduced by the author by adding the new parameter:

\section{$T(n)=p /(1-p * \bmod (r, \operatorname{round}(1 / p))+\operatorname{sqrt}(\operatorname{dist} / 100)$}

Where "dist" is the distance between the "to be elected $\mathrm{CH}$ " and the Base Station.

\section{Simulation \& Result}

For this research MATLAB as simulation Environment has been used simulating the code [12] for original LEACH.

Author maximizes the no. of cycles the network functions for in order to increase the throughput of network.

Author used a network comprising of 100 nodes deployed in a random fashion and have kept the maximum number of cycles as 1500 .

The following data was collected after running the code multiple times:
Table 1 Comparison of results

\begin{tabular}{|l|l|}
\hline \multicolumn{2}{|c|}{ Total Number of Cycles of Network } \\
\hline Original Formula & Proposed Formula \\
\hline 939 & 899 \\
\hline 981 & 956 \\
\hline 960 & 1125 \\
\hline 998 & 1097 \\
\hline 1011 & 1068 \\
\hline 971 & 1007 \\
\hline 921 & 1113 \\
\hline
\end{tabular}

\subsection{Conclusion}

It was found that there is an increase of almost 7 to 15 percent in the total number of cycles, in most of the results.

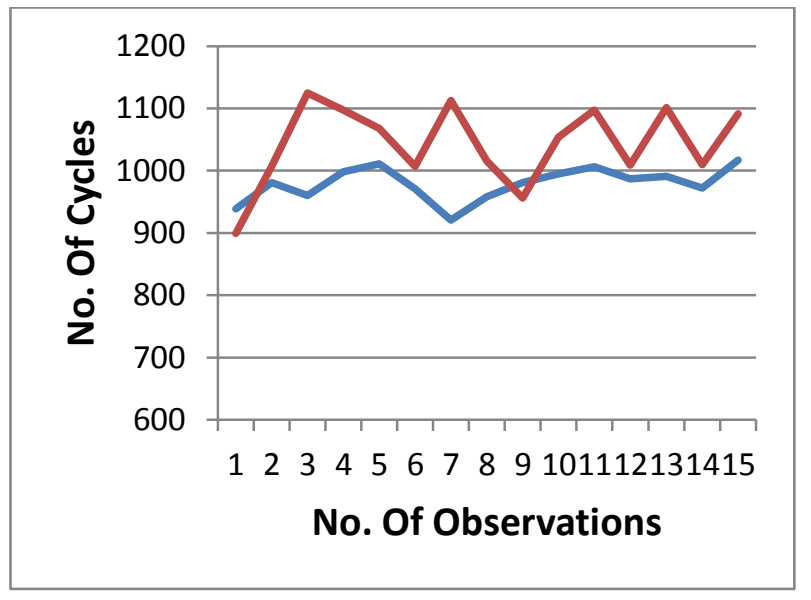

Fig.5 No. of cycles Vs No. Of Observations

This graph shows how proposed version of the protocol is in comparison to the original leach protocol.

The Blue line indicates results for the original formula while the Brown one indicates the results for proposed version of the original formula.

\section{REFERENCES}

[1] M. Bani Yassein, A. Al-zou'bi, Y. Khamayseh, W. Mardini. Application-Specific Protocol Architectures for Wireless Networks. International Journal of Digital Content Technology and its Applications, Volume 3, Number 2, June 2009.

[2] Baiping Li1, Xiaoqin Zhang. Research \& Improvement of LEACH Protocol for Wireless Sensor Network. International Conference on Information Engineering, 2012.

[3] Dilip Kumar, Trilok C. Aseri, R.B. Patel. EEHC: Energy efficient heterogeneous clustered scheme for wireless sensor networks. Computer Communications 32 (2009) 662-667.

[4] Zhu Yonga, Qing Peia. An Energy-Efficient Clustering Routing Algorithm Based on Distance and Residual Energy for Wireless Sensor Networks. International Workshop on Information and Electronics Engineering 
(IWIEE),2012.

[5] Jiguo Yua, Yingying Qia, Guanghui Wangb, Xin Gua. A cluster-based routing protocol for wireless sensor networks with nonuniform node distribution. International Journal of Electronics and Communications.

[6] SamerA. B.Awwad, CheeKyun Ng.NorK, Noordin Mohd, Fadlee A. Rasid. Cluster Based Routing Protocol for Mobile Nodes in Wireless Sensor Network Wireless Pers Commun (2011) 61:251-281.

[7] Vinh Tran-Quang, Takumi Miyoshi. A novel gossip based sensing coverage algorithm for dense wireless sensor networks Computer Networks 53 (2009) 22752287

[8] Er. Pragati, Dr. Rajender Nath. Performance Evaluation of AODV, LEACH \& TORA Protocols through Simulation. International Journal of Advanced Research in Computer Science \& Software Engineering

[9] M.Shankar, Dr.M.Sridar, Dr.M.Rajani. Performance Evaluation of LEACH Protocol in Wireless
NetworkInternational Journal of Scientific \& Engineering Research, Volume 3, Issue 1, January-2012.

[10] Wendi Rabiner Heinzelman, Anantha Chandrakasan, and Hari Balakrishnan. Energy-Efficient Communication Protocol for Wireless Microsensor Networks. Proceedings of the 33rd Hawaii International Conference on System Sciences, USA, pp.8020-8029 January 2000

[11] Wendi Rabiner Heinzelman, Anantha Chandrakasan, and Hari Balakrishnan. An Application-Specific Protocol Architecture for Wireless Microsensor Networks IEEE TRANSACTIONS ON WIRELESS COMMUNICATIONS, VOL. 1, NO. 4, OCTOBER 2002.

[12] W.R.Heinzelman, A.P.Chandrakasan and H.Balakrishnan, "An application-specific protocol architecture for wireless microsensor networks" IEEE Transactions on Wireless Communications, 1(4):660670,2002Mohammad Hossein Homaei Homaei@wsnlab.ir \& Farhadi@wsnlab.ir \& Ranjbaran@wsnlab.ir www.wsnlab.ir 